\title{
Use of systems change and health information technology to integrate comprehensive tobacco cessation services in a statewide system for delivery of healthcare
}

\author{
Sarah Moody-Thomas ${ }^{1^{*}}$, Michael D. Celestin Jr. ${ }^{1}$, Ronald Horswell ${ }^{2}$ \\ ${ }^{1}$ School of Public Health, Louisiana State University Health Sciences Center, New Orleans, USA; \\ *Corresponding Author: sthoma@1suhsc.edu \\ ${ }^{2}$ Pennington Biomedical Research Center, Louisiana State University System, Baton Rouge, USA
}

Received 27 October 2012; revised 28 November 2012; accepted 7 December 2012

\begin{abstract}
Despite the availability of effective treatments and recommendations for systems change, full application of the US Public Health Service clinical practice guideline for the treatment of tobacco use is seldom achieved. The present report describes a comprehensive, structured approach used to implement the guideline and to integrate evidence-based cessation services into a system for delivery of health care. The PRECEDE-PROCEDE model and systems strategies were employed to design and implement the Tobacco Control Initiative ( $\mathrm{TCl}$ ), which provides evidence-based cessation services for the patients of a statewide public hospital system. For the TCl, multi-level assessments, pilot programs, electronic data collection, and performance feedback were needed to produce system-wide changes in workflow and in the quality of care for tobacco users. Although there are advances in health information technology (HIT), systems approaches are required for responding effectively to the Health Information Technology for Economic and Clinical Health (HITECH) Act and to standards governing use of electronic data related to treatment of tobacco use and dependence.
\end{abstract}

Keywords: Tobacco Cessation; Health Care Delivery System; Systems Change

\section{INTRODUCTION}

Tobacco use remains a public health challenge. As the most preventable cause of death and illness, it is the primary risk factor for cardiovascular disease [1] and the leading cause of death in the U.S. [2]. It also undermines management of chronic conditions such as diabetes, asthma and HIV/AIDS [3]. Over the past 60 years, effecttive treatments and policies have decreased tobacco use in the US; there are now fewer current smokers and more never smokers. However, the proportion of former smokers has remained steady for several decades [4]. Those who continue to smoke are more likely to have limited wealth, low educational attainment, and to represent racial/ethnic minorities (e.g., African Americans, Hispanics, and Native Americans) [5]. The burden of tobacco use is evident in the poor health outcomes in these groups, making it imperative to find ways to increase the use of effective treatments.

Health care delivery systems reach large populations of tobacco users. Of the 45 million adult smokers in the US, 70\% visit a health care provider each year [6]; such contacts provide opportunities for cessation. Even brief advice to quit given by a health care provider increases cessation rates [7], and the effect of provider advice is stronger for smokers on Medicaid and uninsured groups of smokers than for those with private insurance [8].

The US Public Health Service (USPHS) clinical practice guideline (CPG) for treating tobacco use and dependence, initially produced in 1996, primarily recommends medication and counseling. An updated guideline, issued in 2000 , encourages health care delivery systems to promote tobacco cessation and to ensure that smokers are identified and engaged during clinical encounters. The 5A's clinical protocol (Ask, Advise, Assess, Assist, and Arrange) is complemented by six system-level strategies to facilitate integration of evidence-based treatments of tobacco use into routine health care practices. The strategies include: 1) identification of tobacco users; 2) provision of education, resources, and performance feedback to health care clinicians; 3 ) use of dedicated staff to coordinate and promote delivery of treat- 
ment; 4) adoption of policies to create supportive services; 5) reduction of patients' out-of-pocket costs for behavioral and pharmacologic treatments; and 6) reimbursement of clinicians delivering cessation treatments [9]. Initiatives sponsored by the Robert Wood Johnson Foundation heightened awareness of the issue and produced evidence supporting the feasibility and effectiveness of changing aspects of health care practice to support treatment of tobacco dependence (e.g., methods to identify smokers in primary care office settings and fax referrals to treatment) [10-12].

Despite evidence of the effectiveness of a "systems approach" [11], widespread implementation of the USPHS CPG continues to lag [13]. Health care systems often lack the resources, infrastructure, and organizational culture needed to overcome barriers to change [14]. Few studies have examined implementation of the CPG for treatment of tobacco use in health care systems serving the most medically vulnerable [15-17].

In view of the fact that Louisiana's smoking rate in $2001(24.6 \%)$ exceeded that of the nation $(22.9 \%)$ [18], the Louisiana state legislature enacted an excise tax on cigarettes in 2002. A portion of the proceeds were dedicated to the provision of cessation services to patients of the state's public hospital system, which is among the largest "safety-net" organizations for health care delivery in the country. Comprised of ten sites, this integrated state-run provider group is operated by the Louisiana State University (LSU) System (see Figure 1).

In the LSU System, every resident of the state is entitled to receive care in any site without regard for enrollment or eligibility criteria. Louisiana's most medically vulnerable are cared for each year, with more than 70,000 admissions, and nearly 1.4 million outpatient and emergency department visits for 400,000 state residents. Patients are $52 \%$ female, 49 years of age on average, 77\% African American, and 49\% uninsured. Facilities

\section{LSU HEALTH SYSTEM SITES}

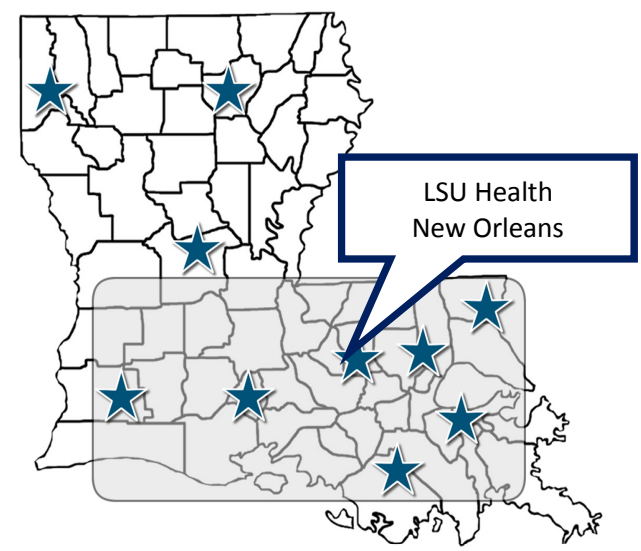

Figure 1. LSU health New Orleans system sites. also serve as training sites for medical and allied health professionals.

The disease management program of the LSU Health New Orleans (LSUH-NO) system, representing 7 of the 10 public hospitals, was initiated to monitor modifiable risk factors and coordinate services to improve health outcomes of patients with chronic conditions. With its existing organizational structure, delivery of proto-coldriven clinical care to defined populations, information technology capacity for patient tracking, and the possibility of performance feedback and program evaluation, this program was identified as an access point for the integration of cessation services into routine health care delivery. Details of this program are described elsewhere [19]. Electronic health records and health information technology (HIT) hold promise for facilitating implementation of the CPG [20]. At the time the disease management program was initiated neither the electronic identification of tobacco users nor documentation of provider intervention was included in the existing infrastructure.

The present report describes the development, implementation, and evaluation of the Tobacco Control Initiative (TCI), a multifaceted program employing system strategies recommended in the USPHS CPG and HIT to integrate evidence-based cessation services into a public health care network.

\section{METHODS}

\subsection{Conceptual Framework}

Systems' thinking [21] was utilized to comprehend the complexity of the healthcare delivery system, interpret multiple layers of assessments conducted among stakeholders, and apply findings toward development of objectives, strategies, and activities, all organized within the structure of the PRECEDE-PROCEED model. This model allows analysis of a situation and design of a health program. It also provides a structure for assessing health and quality of life needs and for designing, implementing, and evaluating health promotion and other public health programs to meet those needs. The model, which directs attention to outcomes rather than to inputs, guides planners through a process that starts with desired outcomes and identifies strategies for achieving objectives. A fundamental characteristic of the model is the participation of its intended audience [22].

\subsection{Program Development}

Formative research was conducted to 1) delineate the organizational structure, processes of care, and IT capacities within the system; 2) determine provider awareness and application of the USPHS CPG and the feasibility of using recommended systems strategies; 3) identify 
barriers to CPG implementation; 4) characterize prevalence and patterns of tobacco use and treatment preferences among patients; and 5) perform a pilot implementation of evidence-based cessation services in a clinical setting. In 2003, a baseline site survey was conducted. The survey was adapted from a series of assessments conducted by the American Association of Health Plans (AAHP) a comprehensive and widely used appraisal of tobacco-related interventions in health care systems [23]. At each site, the survey was administered to representatives from relevant departments [24]. This study received exempt research status from the LSU Health Sciences Center Internal Review Board (IRB).

In 2004, a baseline patient survey characterized prevalence and patterns of tobacco use among patients. A scheme involving stratified two-stage cluster sampling was used to recruit 770 patients to complete face-to-face surveys during visits to targeted primary care clinics [unpublished data]. The target population for this study was defined as adults who use LSU primary care clinics as their principal source of health care. This study was approved by the LSU Health Sciences Center IRB.

Also in 2004, a pilot evaluation of the cessation program was conducted in the dental clinic of one of the network sites. The pilot study was designed to identify and address system barriers to implementation of the clinical protocol based on the 5A's. The pilot program integrated tobacco cessation services into existing processes and included: 1) provider and staff training; 2) identification and assessment of tobacco users; 3) referral to cessation services; 4) delivery of pharmacotherapy and behavioral counseling; and, 5) provision of telephone follow-ups. After data from the assessments and the pilot were compiled, meetings were held at each site to introduce the TCI as a standardized, system-wide intervention for tobacco cessation designed to integrate cessation services into routine health care delivery, present roles and responsibilities of TCI and site staff, and identify a tobacco team leader and potential multi-disciplinary team members.

\subsection{Program Implementation}

In conjunction with the systems strategies delineated in the $\mathrm{CPG}$, a pragmatic approach to implementing a tobacco cessation program in a network setting guided implementation of the TCI [25]. A centralized management team was established to conduct site visits, develop program content, hire cessation coordinators, train site clinicians and staff, and create processes for data collection and reporting.

\subsection{Program Evaluation}

The following measures were used to assess program development and implementation: 1) number of site visits (e.g., presentations at meetings); 2) number of multidisciplinary tobacco control teams formed; 3) development of a system-wide, standardized process of care (paper and electronic, for in-patients, out-patients, and emergency) for identification, documentation, and treatment; 4) number of dedicated staff hired for each hospital; 5) development of data sources (e.g., tobacco registry, referrals, and service delivery databases); 6) number of treatment options established (self-help, group counseling, and medication) at each hospital; and 7) number of clinicians trained in evidence-based practice and process of care.

Evaluation of the impact of the TCI was guided by the document, "A National Blueprint for Disseminating and Implementing Evidence-Based Clinical and Community Strategies to Promote Tobacco-Use Cessation," presented at the National Conference on Tobacco or Health in 2002 [26]. The blueprint was designed to direct activties related to tobacco cessation and to ensure that effective, multi-level efforts support cessation across health care plans and community organizations. The overall goal of the TCI is to implement and evaluate multi-level tobacco cessation services in all hospitals. To do so, objectives for system, provider, and tobacco users were set. The TCI adapted objectives recommended for the four levels of intervention proposed in the blueprint: clinician, system, tobacco user, and evaluation (see Table 1).

Patient responses to the ASK, ADVISE, ASSESS, and ASSIST queries were entered by LSUH-NO clinical personnel into CLIQ (CLinical InQuiry), the LSUH-NO electronic medical record. Patient data on ARRANGED cessation services and follow-up were entered by TCI site personnel into the LSUH-NO Cessation Management and Evaluation Database (CMED), a relational database used to record and manage tobacco cessation activity. Data from CLIQ and CMED were migrated to the LSUH-NO Disease Management \& Evaluation Database (DMED) and merged to calculate rates of provider performance on a monthly basis. Longitudinal data on patient smoking status allowed classifying patients as "smoked in the past 30 days" or "smoked in the past 12 months" and were used to derive quit attempts, sustained quit rates, and relapse rates. Data were also available to identify patients diagnosed with chronic diseases (e.g. asthma, diabetes, HIV-AIDS).

\section{RESULTS}

\subsection{Formative Research}

Site assessments indicated respondents were generally not aware of the CPG and thus had not implemented it. Barriers to implementation were found to be lack of time, space, and skills to engage tobacco users, lack of a consistent place to refer patients for treatment, treatment 
Table 1. TCI objectives, 2003.

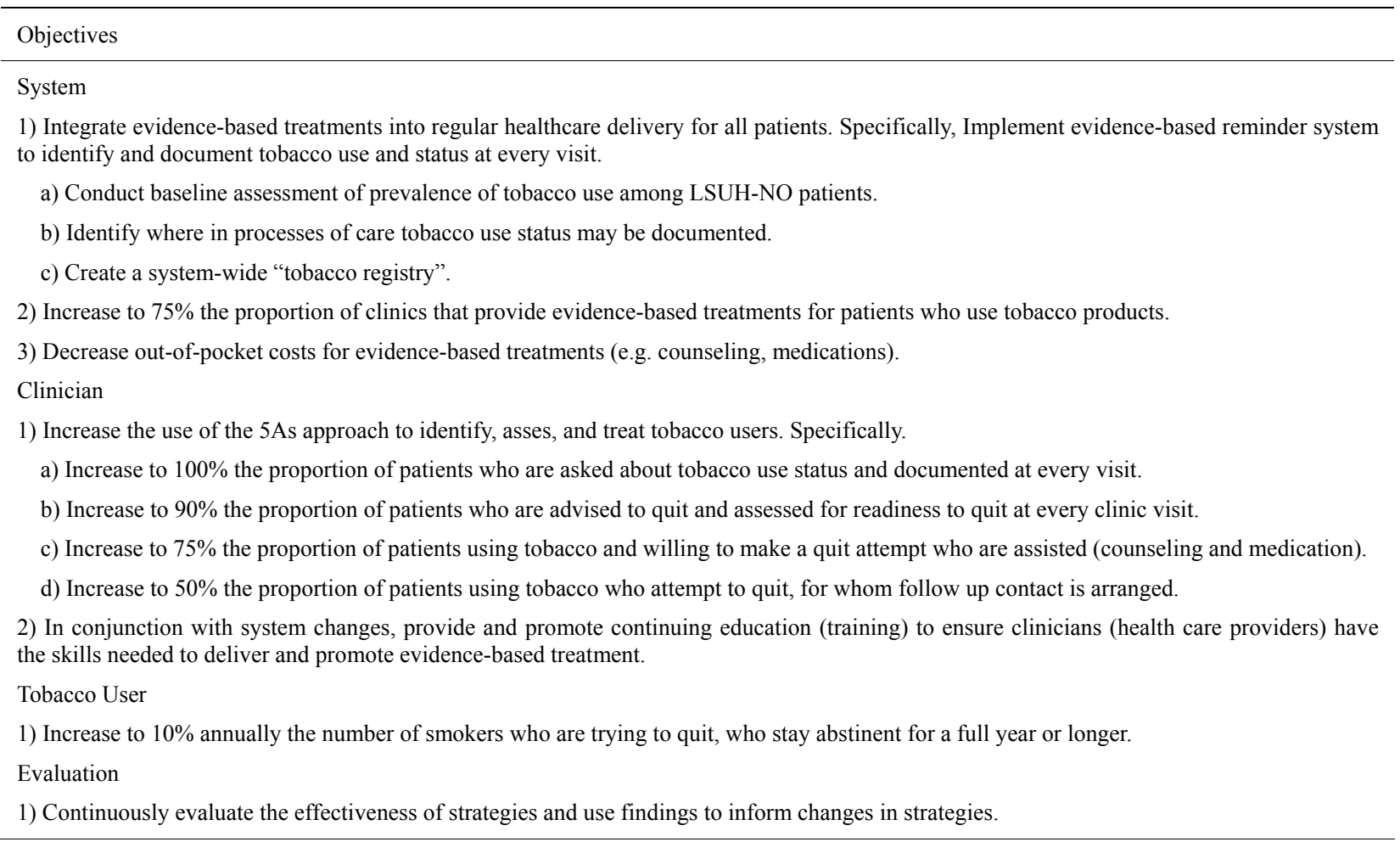

costs, and patients' motivation to quit. All sites expressed a need for personnel dedicated to the delivery and coordination of care for tobacco users. However, there was no system-wide policy delineating a protocol for the treatment of tobacco use.

An estimated $26 \%$ of patients were identified as current smokers, two thirds of which smoked their first cigarette within 30 minutes of awakening; nearly all reported wanting to quit. Preferred treatments, if cost was not an issue, included cessation classes and pharmacotherapy (e.g., nicotine replacement therapy, Zyban). Although more than one third indicated they would call a quit line for assistance, nearly the same number would use non-evidence based treatments (e.g., acupuncture, herbs, and hypnosis); nearly one quarter would get information from the internet.

Results of the pilot at the dental clinic indicated: 1) evidence-based cessation services could be integrated into routine processes of care; 2) in lieu of an electronic health record, the use of standardized forms facilitated process implementation and helped to identify problems therein; 3) provider and staff training were an essential component of service implementation; 4) dedicated staff were needed to ensure patient referral and participation and delivery of cessation services; 5) barriers to patient participation in behavioral counseling had to be addressed; and, 6) issues related to dispensing medication and capacity of on-site pharmacies warranted attention.

\subsection{Program Evaluation}

TCI formed seven site-specific tobacco teams to discuss program status and generate process improvement strategies. These teams created synergy among multidisciplinary team members, coordinated program implementation, and participated in interviewing and hiring TCI staff. A standardized process for identifying and treating tobacco users was developed in conjunction with the tobacco teams from all LSUH-NO sites (see Figure 2).

In regions across the state, the TCI (staff, services, and referral systems) was implemented in four phases, based upon site readiness and personnel recruitment efforts.

The use of HIT evolved as the system's capabilities developed. Prior to 2004, all documentation was made in clinical charts and required manual verification. Further, the charts did not prompt adherence to the clinical protocol. In 2004, the TCI introduced a paper referral form to document assessment of tobacco use, cessation medication consults, and referral to on-site group behavioral counseling. Forms were collected and entered by TCI staff into CMED for treatment follow-up and provider feedback.

In 2005, an electronic Tobacco Registry was created by asking all patients "Have you used tobacco in the past 30 days?" during the financial certification that is required every 180 days. The registry provided a denominator 


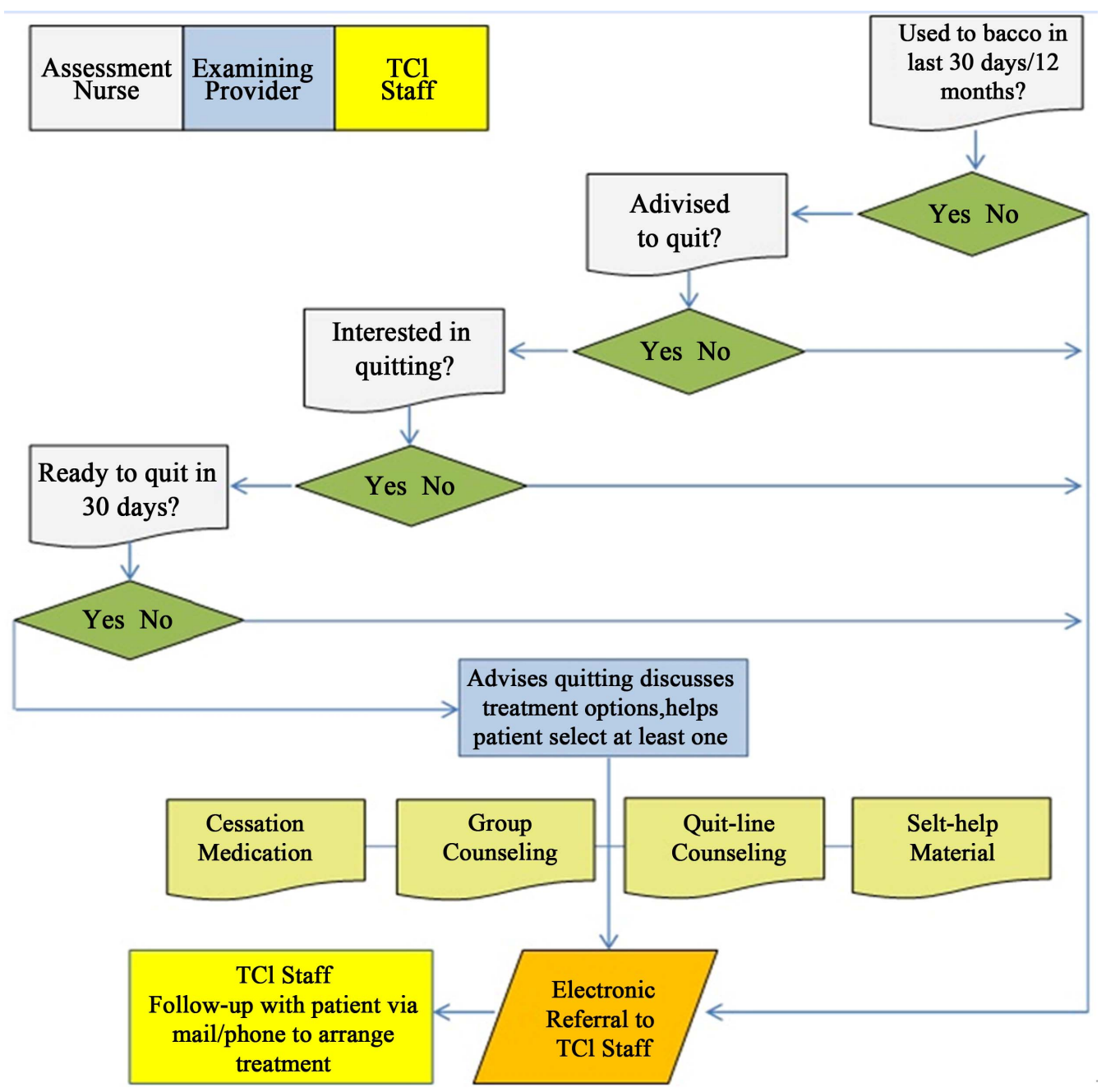

Figure 2. Flowchart for outpatient documentation and treatment of tobacco use.

of all smokers, essential for analytic and program evaluation purposes. Data were stored in the administrative database and paired with CMED and the DMED. DMED captured administrative data, such as patient encounters, for each chronic illness and provided data to assess quality of care, access to care, and health outcomes for all LSUH-NO disease management programs.

Although the Tobacco Registry data were useful, the status of patients' tobacco use was not available to clinicians at the point of care. In 2007, the same question was included in CLIQ. This provided an opportunity to access information in the clinical setting regarding smoking status, as well as to prompt the reassessment and treatment of smokers, every 90 days. In 2008, the Plan, Do, Check, Act (PDCA) model was used to pilot a standardized process for documenting use of the 5 A's protocol. In 2009, LSUH-NO deployed a revised CLIQ screening system that included all 5 A's as well as electronic referral to evidence-based cessation services. This HIT upgrade made it possible to achieve programmatic goals. In
2010, LSUH-NO again revised the CLIQ screening to identify smokers interested in quitting, in addition to those ready to quit in 30 days, and required that self-help information be given to all smokers instead of including as a choice for treatment. See Figure 3 for a timeline of events.

TCI field staff based at each site was hired to accomplish the program goals. Essential functions included facilitating behavioral counseling, telephone follow-up calls, patient recruitment, internal marketing to patients and providers, convening tobacco teams, and data collection and reporting. The staff members were required to participate in weekly conference calls that highlighted best practices, protocol adherence, and opportunities for process improvement. Comprehensive training and development activities were provided to enhance the skill levels of TCI field staff. Training included program orientation, computer-based tobacco control education, behavioral counseling curriculum, motivational enhancement therapy, social marketing, team building, and data 


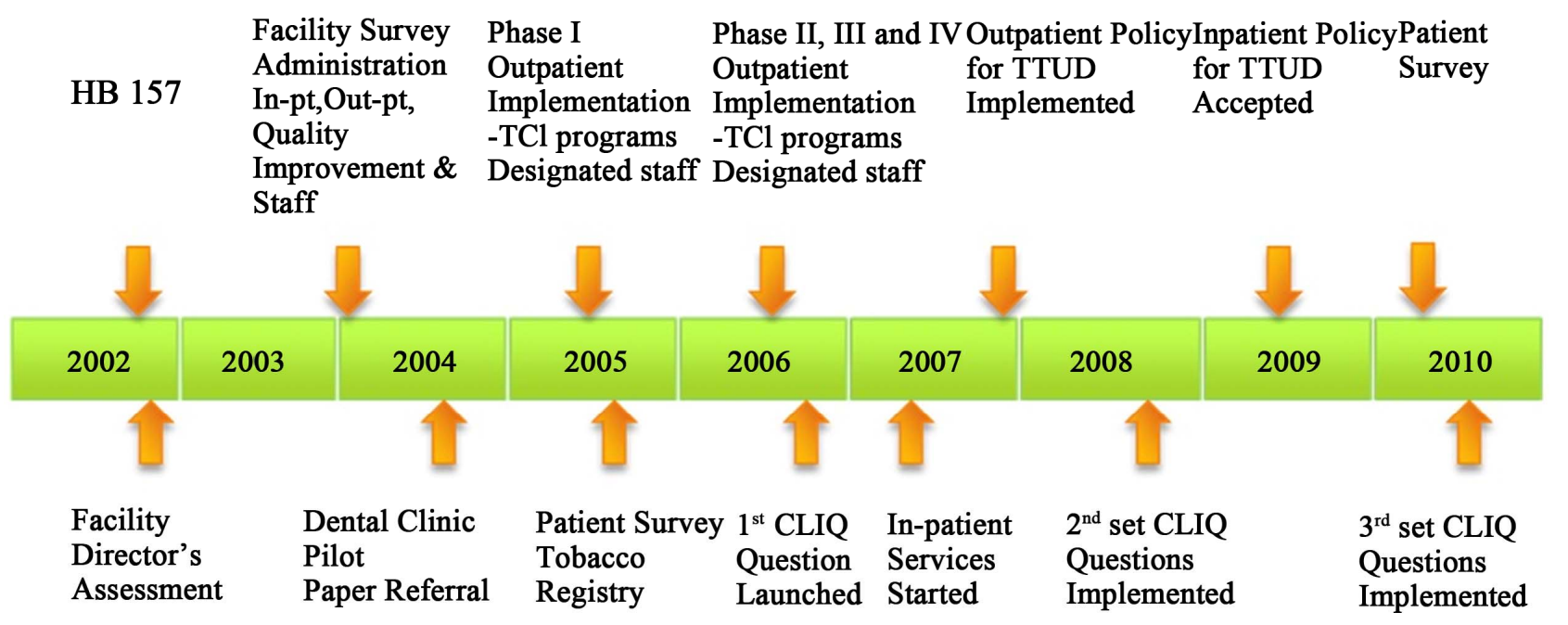

Figure 3. TCI timeline of events.

base management.

Provider engagement regarding tobacco use was documented on elements of the 5 A's protocol (see Figure 4).

Arrange, defined as provider referral to cessation specialists for receipt of selected services, reached 100\% because it was an electronic referral system via CliQ.

TCI staff provided face-to-face in-service training on identification and treatment of tobacco users, referral procedures, and available evidence-based cessation resources for 210 doctors in 2006. In 2007, the TCI linked the University of Wisconsin's web-based training on Treatment of Tobacco Use and Dependence (TTUD) to the existing LSUH-NO provider e-learning system. With face-to-face in-service training and the web-based course, the number of clinicians trained increased to 934 in 2007 , 1445 in 2008, and 1472 in 2009. In 2008, the LSUH-NO adopted a policy requiring completion of the online course by all nurses and other staff. Documentation of completion was entered into their personnel files.

Quit rates (defined as quit after 90 days and sustained quit after another 90 days and calculated electronically using (liQ) increased from $5.0 \%$ in 2008 to $9.6 \%$ in 2009. Also in 2009, chronically ill patients (except those with HIV) reported lower 30-day (26\%) prevalence rates than the general patient population $(29 \%)$. Asthma patients were least likely to report smoking (18\%) and had a higher quit rate $(15 \%)$ compared to other groups ( $p>$ $0.05)$. In contrast, HIV patients were more likely to report smoking (43\%) and had the lowest quit rate $(6 \%)(\mathrm{p}$ $<0.05)$.

\section{DISCUSSION}

This report describes how the PRECEED/PROCEED planning and delivery model [22] was used in conjunction with systems thinking [21] to design and implement comprehensive, evidence-based tobacco cessation services in a healthcare system serving the medically vulnerable. Multilevel assessments, pilot projects, and rapid learning studies provide information about patient treatment preferences and organizational structures affecting CPG implementation. Electronic identification of all tobacco users prompts routine screening, assessment, and treatment and creates a data source for determining population-based smoking prevalence, program evaluation, and performance feedback. This approach ensures stakeholder involvement in the development of workflow and treatment protocols and fidelity of protocol implementation, delivers performance feedback for administrators and providers, and maximizes the likelihood of sustainable integration of cessation services in the healthcare setting [27].

CPGs are designed using existing evidence and, when implemented, are expected to improve patterns of care and health outcomes [28]. CPG implementation, however, is difficult to achieve due to the complex intervenetions prescribed and to the complex environments in which it is to be implemented [29,30]. With a systems approach, the TCI created synergy of implementation strategies and built the infrastructure for CPG implementation. This process is referred to as "organizational innovation" [11]. Although there are examples of evidence-based cessation services within health care settings [31-34], none describe development and implementation of a comprehensive, standardized, systemwide program for tobacco treatment $[6,13]$.

Rigotti emphasizes the need for a comprehensive tobacco care management system by stating, "if tobacco treatment is to be integrated into the rapidly evolving US health care system, we must create a comprehensive care management system similar to that used for other chronic diseases" [6,33,35-36]. The TCI accessed the LSUH-NO 


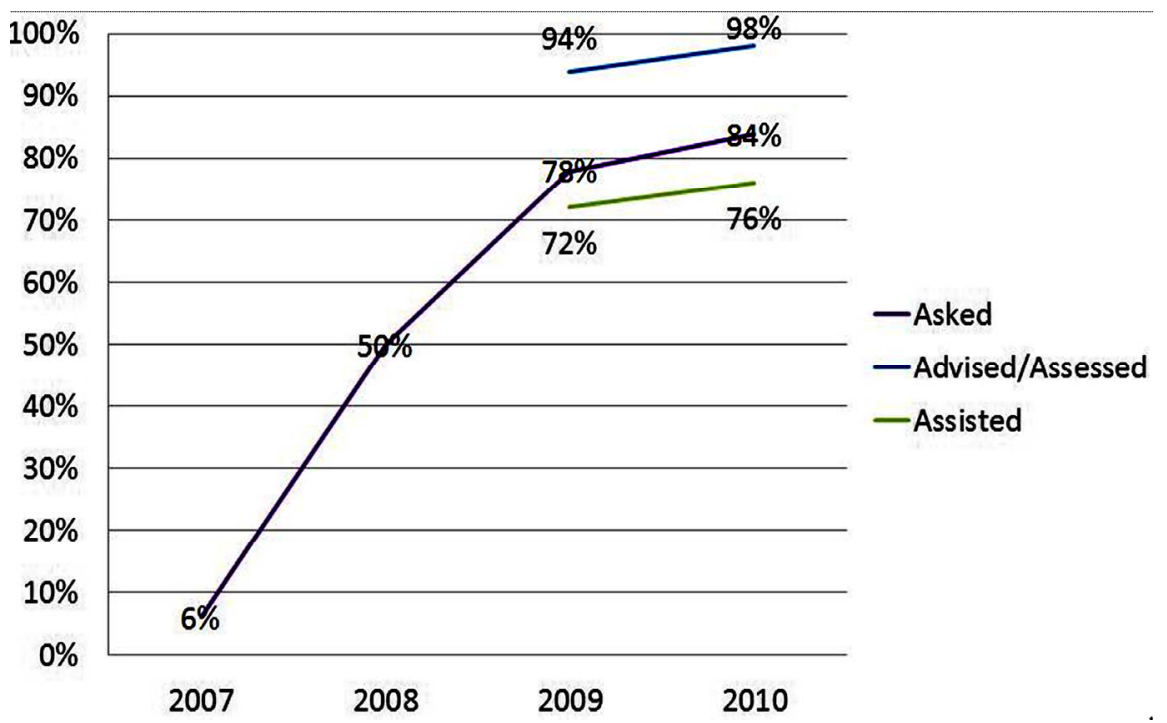

Figure 4. 5 A's intervention (2007-2010).

system through its existing Disease Management Program. The CPG offered the advantage of evidence-based tactics to guide the implementation process. The TCI, a comprehensive tobacco management system, was created to implement the CPG. The process included identification of all eligible patients, direct outreach to the specific population, central coordination of treatment sites, and system-wide, standardized interventions. Although these components are recommended [36], other disease management programs in the LSUH-NO system do not offer them.

\section{CONCLUSIONS}

The TCI program is an inclusive tobacco cessation intervention. Its structure and support allow the program to identify sub-groups of patients (e.g., diabetic smokers) and specific patient behaviors (relapse among recent quitters) to be targeted with more focused interventions. This coupling of program structure with the capabilities of modern electronic medical records should lead to additional tobacco control innovations.

The Health Information Technology for Economic and Clinical Health (HITECH) Act, which was part of the American Recovery and Reinvestment Act of 2009, is designed to promote the adoption and meaningful use of HIT. It incentivizes the adoption of electronic health records and sets criteria for minimum data requirements for comparability and multi-site sharing [37]. While electronic health records can produce large quantities of data, solutions to real-world impediments to change and innovation come through systems thinking [38]. Identification of the interrelated parts of the system is an initial process. The interventionist must also determine the order in which to initiate change strategies within and among components and the policies needed to reinforce and sustain change. Innovation does not translate to improved population health without widespread and sustained uptake of effective changes. Although real-world constraints persist, the LSU Health System has experienced organizational innovation, and the infrastructure is in place for more formal investigations in regard to ideal time, places, approaches, and methods for dissemination and implementation [27].

The TCI highlights the promise of technology and appropriate policies to improve care in a delivery system serving the medically vulnerable. The program illustrates a partnership between medicine and public health, in which basic, clinical, behavioral, and systems sciences converge to make treatment of tobacco use standard care.

\section{REFERENCES}

[1] Centers for Disease Control and Prevention (US), National Center for Chronic Disease Prevention and Health Promotion (US), Office on Smoking and Health (US) (2010) How tobacco smoke causes disease: The biology and behavioral basis for smoking-attributable disease. A report of the surgeon general. Centers for Disease Control and Prevention (US), Atlanta (GA).

[2] Centers for Disease Control and Prevention (CDC) (2008) Smoking-attributable mortality, years of potential life lost, and productivity losses-United States, 2000-2004. Morbidity and Mortality Weekly Report (MMWR), 57, 12261268.

[3] Office of the Surgeon General (US), Office on Smoking and Health (US) (2004) The health consequences of smoking: A report of the surgeon general. Centers for Disease Control and Prevention (US), Atlanta (GA).

[4] Centers for Disease Control and Prevention (CDC) (2009) State-specific smoking-Attributable mortality and years 
of potential life lost-United States, 2000-2004. Morbidity and Mortality Weekly Report (MMWR), 58, 29-33.

[5] Centers for Disease Control and Prevention (CDC) (2011) Vital signs: Current cigarette smoking among adults aged $\geq 18$ years-United States, 2005-2010. Morbidity and Mortality Weekly Report (MMWR), 60, 1207-1212.

[6] Curry, S.J., Sporer, A.K., Pugach, O., Campbell, R.T. and Emery S. (2007) Use of tobacco cessation treatments among young adult smokers: 2005 national health interview survey. American Journal of Public Health, 97, 146461469. doi:10.2105/AJPH.2006.103788

[7] Stead, L.F., Bergson, G. and Lancaster, T. (2008) Physician advice for smoking cessation. Cochrane Database of Systematic Reviews, 2, Article ID: CD000165. doi:10.1002/14651858.CD000165.pub3

[8] Cokkinides, V.E., Ward, E., Jemal, A. and Thun, M.J. (2005) Under-use of smoking cessation treatments: Results from the National Health Interview Survey, 2000. American Journal of Preventive Medicine, 28, 119-122. doi:10.1016/j.amepre.2004.09.007

[9] Fiore M.C., Bailey W.C., Cohen S.J., Dorfman, S.F., Goldstein, M.G., Gritz, E.R., et al. (2000) Treating tobacco use and dependence. Clinical practice guideline. Department of Health and Human Services, Rockville.

[10] Orleans, C.T. (1998) Challenges and opportunities for tobacco control: The robert wood johnson foundation agenda. Tobacco Control, 7, S8-S10. doi:10.1136/tc.7.2008.S8

[11] Curry, S.J. (2000) Organizational interventions to encourage guideline implementation. CHEST, 118, 40S-46S. doi:10.1378/chest.118.2 suppl.40S

[12] Curry, S.J., Orleans, C.T., Keller, P. and Fiore, M. (2006) Promoting smoking cessation in the healthcare environment. American Journal of Preventive Medicine, 31, 269272. doi:10.1016/j.amepre.2006.05.003

[13] Fiore M.C., Jaén C.R., Baker T.B., Bailey, W.C., Benowitz, N.L., Curry, S.J., et al. (2008) Treating tobacco use and dependence: 2008 update. Clinical Practice Guideline, US Department of Health and Human Services, Rockville.

[14] Moolchen, E.T., Fagan, P., Fernander, A.F., Velicer, W.F., Hayward, M.D., King, G., et al. (2007) Addressing tobacco related health disparities. Addiction, 102, 30-42. doi:10.1111/j.1360-0443.2007.01953.x

[15] Zapka, J.G., White, M.J., Reed, G., Ockene, J.K., List, E., Pbert, L., Jolicoeur, D. and Reiff-Hekking, S. (2005) Organizational systems to support publicly funded tobacco treatment services. American Journal of Preventive Medicine, 28, 338-345. doi:10.1016/j.amepre.2005.01.008

[16] Santos, L., Braun, K., Aea, K. and Shearer, L. (2008) Institutionalizing a comprehensive tobacco cessation protocol in an indigenous health system: Lessons learned. Progress in Community Health Partnerships, 2, 279-289. doi:10.1353/cpr.0.0038

[17] Foley, K.L., Pockey, J.R., Helme, D.W., Song, E.Y., Steward, K., Jones, C., Spangler, J.G. and Sutfin, E.L. (2012) Integrating evidence-based tobacco cessation interventions in free medical clinics: Opportunities and challenges. Health Promotion Practice, 13, 687-695.

\section{doi: $10.1177 / 1524839911433465$}

[18] Centers for Disease Control (CDC) (2001) Behavioral risk factor surveillance system survey data. US Department of Health and Human Services, Atlanta

[19] Horswell, R., Butler, M.K., Kaiser, M., Moody-Thomas, S., McNabb, S., Besse, J., et al. (2008) Disease management programs for the underserved. Disease Management, 11, 145-152. doi:10.1089/dis.2007.0011

[20] Boyle R., Solberg L. and Fiore, M. (2011) Use of electronic health records to support smoking cessation. Cochrane Database of Systematic Reviews, 12, Article ID: CD008743. doi:10.1002/14651858.CD008743.pub2

[21] National Cancer Institute. (2007) Greater than the sum: Systems thinking in tobacco control: Tobacco control monograph No. 18. US Department of Health and Human Services, Bethesda.

[22] Green, L.W. and Kreuter, M.W. (2005). Health program planning: An educational and ecological approach. McGraw-Hill Higher Education, New York.

[23] McPhillips-Tangum, C., Rehm, B., Carreon, R., Erceg, C.M. and Bocchino, C. (2006) Addressing tobacco in managed care: Results of the 2003 survey. Preventing Chronic Disease, 3, 1-11.

[24] Thomas, S.M., Horswell, R., Celestin, M.D., Dellinger, A.B., Kaiser, M. and Butler, M. (2011) Awareness and implementation of the 2000 United States public health service Tobacco dependence treatment guideline in a public hospital system. Population Health Management, 4, 79-85. doi:10.1089/pop.2010.0004

[25] Krejci, R. (2000) Tobacco cessation program implementation-From plans to reality: Skill building workshopnetwork model. Tobacco Control, 9, i33-i36. doi:10.1136/tc.9.suppl 1.i33

[26] National Tobacco Cessation Collaborative (2002) A national blueprint for disseminating and implementing evidence-based clinical and community strategies to promote tobacco-use cessation. http://www.tobacco-cessation.org/PDFs/blueprint adult.p df

[27] Glasgow, R.E., Vinson, C., Chambers, D., Khoury, M.J., Kaplan, R.M. and Huncer, C. (2012) National institutes of health approaches to dissemination and implementation science: Current and future directions. American Journal of Public Health, 102, 1274-1281. doi:10.2105/AJPH.2012.300755

[28] Institute of Medicine (2001) Crossing the quality chasm: A new health system for the 21 st century. National Academies Press, Washington DC.

[29] Pawson, R., Greenhalgh, T., Harvey, G. and Walshe, K. (2005) Realist review-A new method of systematic review designed for complex policy interventions. Journal of Health Service Research and Policy, 10, 21-34. doi:10.1258/1355819054308530

[30] Shiell, A., Hawe, P. and Gold, L. (2008) Complex interventions or complex systems? Implications for health economic evaluation. British Medical Journal, 336, 12811283. doi:10.1136/bmj.39569.510521.AD

[31] Bentz, C.J., Bayley, K.B., Bonin, K.E., Fleming, L., 
Hollis, J.F., Hunt, J.S., et al. (2007) Provider feedback to improve 5A's tobacco cessation in primary care: A cluster randomized clinical trial. Nicotine and Tobacco Research, 9, 341-349. doi:10.1080/14622200701188828

[32] Mallen, M.J., Blalock, J.A. and Cinciripini, P.M. (2006) Using technology to serve patients and practitioners: A comprehensive tobacco cessation program for cancer patients. Counseling and Psychotherapy Research, 6, 196201. doi:10.1080/14733140600857550

[33] Sherman, S. (2008) A framework for tobacco control: Lessons learned from Veterans Health Administration. British Medical Journal, 336, 1016-1019. doi:10.1136/bmj.39510.805266.BE

[34] Lindholm, C., Adsit, R., Bain, P., Reber, P.M., Brein, T., Redmond, L., Smith, S.S. and Fiore, M.C. (2010) A demonstration project for using the electronic health record to identify and treat tobacco users. Wisconsin Medical Journal, 109, 335-340.
[35] Rigotti, N.A., Munafo', M.R. and Stead, L.F. (2007) Interventions for smoking cessation in hospitalized patients. Cochrane Database of Systematic Reviews, 5, Article ID: CD001837. doi:10.1002/14651858.CD001837.pub3

[36] Rigotti, N.A. (2011) Integrating comprehensive tobacco treatment into the evolving US health care system. Archives of Internal Medicine, 171, 53-55. doi:10.1001/archinternmed.2010.491

[37] Blumenthal, D. and Tavenner M. (2012) The meaningful use regulation for electronic health records. New England Journal of Medicine, 363, 501-504. doi:10.1056/NEJMp1006114

[38] Leishcow, S.J. and Milstein, B. (2006) Systems thinking and modeling for public health practice. American Journal of Public Health, 96, 403-406. doi:10.2105/AJPH.2005.082842 